\title{
A discriminating model to classify the quality of life of asthmatic children
}

\begin{abstract}
Introduction: There isn't universal definition about quality of life. It's a subjective concept, typical of each person, with an important influence of the environment.

Objective: To Offer a measure of the quality of life of the asthmatic minors this might serve to quantify it in three previously defined categories.

Materials and methods: A descriptive transversal analysis in 20 secondary schools in Andalusia for asthmatic minors between 13 and 14 years old. The Paediatric Asthma Quality of Life Questionnaire has been used as the study tool. To build the functions of classification we have used a discriminant analysis.

Results: We offer a classification model for quality of life of the asthmatic child. The model has a high discriminant power. It correctly classifies $91.8 \%$ of the occasions to the asthmatic in one of the three previously defined categories of quality of life (low, medium and high). It is detected that the emotional function is the variable that has more relevance in the prediction of the group of asthmatic children, followed by the symptoms and the limitations in the habitual activities.
\end{abstract}

Keywords: Asthma, adolescents, quality of life, indicator, classification
Research Article

Volume 7 Issue 2 - 2018

\author{
Ana María Sánchez Sánchez,' Francisca \\ Sánchez Sánchez, ${ }^{2}$ David Ruiz-Muñoz ${ }^{3}$ \\ 1,2Universidad Pablo de Olavide, España \\ Junta de Andalucía, España
}

Correspondence: David Ruiz-Muñoz, Junta de Andalucía, Sevilla, Spain, Email darumu@gmail.com

Received: December 13, 2017 | Published: March 02, 2018

\section{Introduction}

The World Health Organization estimates that there are currently 235 million patients with asthma and that it is the most frequent chronic disease in children, although it can be controlled with different prevention and treatment plans adapted to the symptoms of each patient. Its prevalence varies ostensibly in different geographical areas, with an average prevalence of $10 \%$ in Spain. ${ }^{1}$ Quality of life (QL) is a dynamic and interdependent concept, so that when someone is physically affected, it generates an impact on the affective and social aspects. Sometimes patients with apparently similar pulmonary symptoms may have different perceptions of their quality of life due to the different capacities of appreciation, the characteristics of each personality and even lifestyle. The study carried out during 2013, in the Andalusian province of Granada (Spain). The impact of asthma on children is variable, due to the physical changes of age and cognitive development, which influence the child's perception of his illness, affecting various areas of his life, such as physical, psychological, social and school aspects. ${ }^{2}$ QL can be measured through different variables. In our study we will focus on three aspects that express the dimensions of physical, emotional and social, ${ }^{3}$ including in this last dimension certain aspects related to the family, educational and environmental.

Currently it is recommended that the QL be measured from the perspective of the patient, in this case of children suffering from asthma, since they are the ones who can report on how they have been affected in their daily lives, in their social interactions, in their communication and in their emotional state. In addition, there are different studies that show that the assessment of children, immediate caregivers and the medical staff that serves them differ from each other. Children and adolescents report better on their perception of the quality of life related to childhood health (below HRQL) than do their caregivers and sometimes the medical staff, or the parents themselves, who tend to value more negatively the HRQOL; it is therefore a complex concept, with a multidimensional character ${ }^{4}$ and therefore difficult to measure or quantify. One aspect that helps improve the quality of life in asthmatics is the control of the disease itself. In recent years, the definition of the concept of asthma control has been deepened, as a central objective to treat the disease. ${ }^{5,6}$ Although today it has a pharmacological therapy and well prescribed is highly effective, most asthmatics are not well controlled for various reasons derived from: the same disease, the patient, his family, his environment, the staff of health, of the health system and of the community in general. ${ }^{7}$

In addition to the control of asthma, the treatment of the disease has focused on the control and improvement of quality of life, whose measurement has also been perfected in recent years, ${ }^{8}$ having to take into account its complexity as it is very mediated by the individual perception of each patient of his own $\mathrm{CV} .^{9}$ As a consequence of this subjectivity, several facets or dimensions of the life of each individual must be incorporated in the measurement of the $\mathrm{VC}$, as well as considering and weighing the capacity to participate fully in age-appropriate physical, social and psychosocial functions and activities. ${ }^{10-12}$ The effort necessary to overcome these difficulties is compensated by the acquisition of complementary information that is very useful for making clinical decisions about the status of patients and for evaluating the cost-benefit ratio of the available treatments. There are multiple questionnaires to measure HRQOL. In our case we have opted for the use of the "Pediatric Asthma Quality of Life Questionnaire" (PAQLQ) because it is validated in multiple languages and cultures, as it is a questionnaire that, in addition to being reliable and valid in the population of children with asthma, complies with psychometric properties, has been shown to be sensitive to changes and allow minimal fluctuations in the score to be perceived as a significant change in the quality of life of the patient, ${ }^{13,14}$ which favors and gives robustness to the discriminant model obtained in this work 
and the functions or variables that are identified as more influential in the model itself. In this work, two objectives are proposed:

i. Offer a measure of the HRQOL of asthmatic children that serves to quantify it and classify it in one of the three previously defined categories (low, medium and high), through a mathematical function for each of them.

ii. Determine the importance of the variables of the study, for the classification of the individuals in any of the categories in which the quality of life is measured, analyzing if the quality of life is influenced by the gender of the patient.

\section{Materials and methods}

\section{Design and Population}

To carry out the analysis of quality of life in asthmatic children, a cross-sectional descriptive study was carried out, carrying out a simple random stratified sampling, with proportional affixation of all secondary schools in Granada and province. The data collection and analysis was carried out during the year 2013. The educational centers belonged to the province of Granada (public and private) and located both in rural (population under 5,000 inhabitants) or urban (population over 5,000 inhabitants). To calculate the optimal sample size, the main variable was considered whether the individual had asthma or not. It is therefore feasible in the study of this population, the knowledge of the proportion of inhabitants who had asthma. This allowed the application of the formula of the optimal sample size for a proportion in simple random stratified sampling. We must specify that when it comes to estimating proportions, we considered the worst case that could be presented when estimating the variance of the stratum population, that is, $\mathrm{Ph}=0.5$ and $\mathrm{Qh}=0.5$ were taken, thus guaranteeing that the sample size obtained is significant whatever the variance of our strata. Initially, 31 secondary schools in the province of Granada were invited to participate and, after the approval of the competent school body, 20 schools participated. The centers they accepted remained representative of the rural and urban strata, and of the public and private spheres. The study was carried out among asthmatic students with an age between 13 and 14 years of age $(n=$ 4,247 ), with the prior consent of the parents or legal guardians. To determine the sample size, the following expression was used:

$$
n=\frac{\sum_{h=1}^{L} W h^{2} \cdot \frac{N h}{N h-1} \cdot \frac{P h Q h}{w h}}{\frac{e^{2}}{k^{2}}+\frac{1}{N^{2}} \sum_{h=1}^{L} \frac{N h^{2}}{N h-1} P h Q h}
$$

$\mathrm{n}$ :Total sample size for the population studied

$$
\begin{aligned}
& \mathrm{L}=\text { Number of strata } \\
& \mathrm{N}=\text { Population size studied } \\
& \mathrm{Nh}=\text { Population size school } \\
& \mathrm{Wh}=\text { Population weight school } \\
& \text { wh= Sampling weight school } \\
& \mathrm{Ph}=\text { Proportion of individuals with asthma by school } \\
& \mathrm{Qh}=\text { Proportion of individuals without asthma by school }
\end{aligned}
$$

$$
\mathrm{e}=0.03 \text { Maximum admissible sample error }
$$$$
\mathrm{k}=1.96 \text {. confidence level coefficient } 95 \% \text {. }
$$

\section{Study variables: Procedures/data collection and management}

In order to determine the functions that represented the type of quality of life, a new variable was defined: "quality of life", with three possible categories (low, medium and high), based on the variable quality of life. In particular, the low quality of life category is defined in a score range between [0to3); the quality of life half between [3to5) and the quality of life high between [5to7]. Three classification variables were used: Symptoms, Limitation and Emotional Function for the classification of an asthmatic child in one of the three categories of quality of life. The research team facilitated the questionnaires to the different schools, and these were distributed by the teaching staff to their students, who completed a questionnaire of knowledge and attitudes towards asthma, in a period of about 45 minutes. The exclusion criteria (students without active asthma) adopted to participate in the questionnaire of quality of life, considering as students with active asthma as such those who had a medical diagnosis of asthma and had asthma symptoms or had used asthma medication (beta 2 and/or inhaled corticosteroids) in the last 12 months, also filled out a questionnaire. The questionnaires were anonymous, and they were identified through a numerical code that was associated to the school, to the classroom of belonging and that identified the sex of the students. The questionnaires were collected by the faculty and returned to the researchers.

\section{Methods used for evaluations}

The questionnaire used to evaluate knowledge and attitudes towards asthma was the Newcastle Asthma Knowledge Questionnaire ${ }^{15}$ (NAKQ), validated in Spanish, and made up of 31 dimensions. ${ }^{16}$ This questionnaire assesses knowledge about symptoms, their triggers and their treatment. It consists of 25 questions with unique answers of true or false and 6 open questions, which are assessed according to the instructions of the same questionnaire, with possible values between 0 and 31 points, depending on the answers. ${ }^{17}$ Right answers rate 1 point and wrong answers 0 points. The total score of the questionnaire is obtained by adding up the points for all questions. The final value ranges between 0 and 31 , with a higher score indicating greater knowledge. The text of the first question implies that the answer must include all of the three main symptoms of asthma, so answers have only been rated as correct when all three were enumerated.

The answer to question six has been rated as correct if the respondent named at least one of the three triggers that are established as possible answers. Questions 10, 11, 21, and 23 have been rated as correct when the respondent has given at least two of the answers that the questionnaire offers as possible answers, as indicated by the scoring rules of the original questionnaire. To measure the quality of life, a specific questionnaire was used to study the quality of life in asthmatic children aged 7 to 17 years, the "Pediatric Asthma Quality of Life Questionnaire"18 (PAQLQ) in its Spanish version, which is widely validated ${ }^{19-21}$ and on which there is considerable evidence in the literature that supports its application in different cultures and languages..$^{22}$ Its score is between the value 1 (worse quality of life) and the value 7 (better overall quality of life), having 3 dimensions: symptoms, limitation of activities and emotional function. The results 
for each dimension were the average of the items in each dimension. The overall quality of life was the average score of the 23 items of the questionnaire.

\section{Analysis}

We did the statistical analysis of the data using IBM SPSS Statistics version 22.0.0. As a normality test, the Kolmogorov-Smirnov test was used because the Shapiro-Wilk test was not applicable given the high sample size (over 50). As a result of the normality test, the multivariate normality of the discriminant variables was verified, this being one of the necessary requirements to be able to apply the statistical technique of discriminant analysis. The contrasts were made taking a level of significance of $5 \%$. For the analysis of the variables, the multivariate statistical technique of Discriminant Analysis was used. This analysis is based on a set of elements that belong to different previously established groups, analyzing the information related to a series of variables, with a double purpose: the first one of explanatory character, when determining the contribution of each variable to the correct classification of each element, and the second with predictive character, when determining the group to which each new element belongs. With this technique we try to determine the importance of the classification variables: Symptoms, Limitation and Emotional Function for the classification of an asthmatic child in any of the three categories of quality of life. Once the values of the variables were known, it allowed us to predict or classify in which of the three quality of life groups each asthmatic child was located.

\section{Results}

The questionnaires of knowledge and attitudes towards asthma were completed by 3,101 students $(73 \%)$ and 226 of them were asthmatic. Table 1 shows the characteristics of asthmatic students, relating them to their gender. In addition, a Chi-square test was carried out to study whether quality of life was related to gender and at $5 \%$ of significance it was found that there was no relationship between these variables $(p=0.167)$, that is, there were no differences in the perception of the quality of life that children have. This result meant that the analysis carried out afterwards were carried out without distinction of gender, as it was not a relevant variable.

Table I Quality of life by gender

\begin{tabular}{lllll}
\hline Gender & $\begin{array}{l}\text { Quality life } \\
\text { low }\end{array}$ & $\begin{array}{l}\text { Quality life } \\
\text { medium }\end{array}$ & $\begin{array}{l}\text { Quality } \\
\text { life high }\end{array}$ & Total \\
\hline Children & $1.1 \%$ & $8.6 \%$ & $36.2 \%$ & $45.90 \%$ \\
Girls & $1.8 \%$ & $15.1 \%$ & $37.3 \%$ & $54.10 \%$ \\
Total & $2.9 \%$ & $23.7 \%$ & $73.5 \%$ & $100.00 \%$ \\
\hline
\end{tabular}

Table 2 shows the results of the application of the Analysis of the Variance to each classification variable, so that it is possible to contrast statistically, for each of them, the equality of means in the three groups. The Lambda coefficient of Wilks collects the ratio of the inter-group and intra-group variations from the corresponding sums of the squares of the deviations of the scores with respect to those of the means. Statistic F collects the test value to perform the contrast indicated above, higher values represent greater differences between groups; "Sig." indicates the level of minimum significance, by which the hypothesis contrasted is rejected. Taking a maximum level of significance of $5 \%$, and making use of the minimum level of significance for each of the variables, we can solve the contrasts of equality hypotheses.
Table 2 Evidence of equality of means of the groups

\begin{tabular}{llll}
\hline Variables & $\begin{array}{l}\text { Lambda } \\
\text { wilks }\end{array}$ & $\mathrm{F}$ & Sig. \\
\hline Symptom & 0.338 & 270.835 & 0.000 \\
Limitation & 0.472 & 154.468 & 0.000 \\
Emotional Function & 0.286 & 343.808 & 0.000
\end{tabular}

In conclusion it is obtained that the variables Symptoms, Limitation and Emotional Function are different for the three quality of life groups. This fact constitutes an indication that the three variables have discriminant power and, therefore, should be considered as such in the analysis. Table 3 indicates the relative weight of each variable in the discriminant function, these values being in absolute value. The Emotional Function variable (0.619) is the most relevant when predicting the group belonging to young asthmatics, followed by Symptoms (0.433) and Limitation (0.181).

The classification functions for the three quality of life groups are:

Low quality of life $=0.188 *$ Symptoms $+2.324 *$ Limitation $+5.912 *$ Function-11.993

Average life quality $=0.489 * \quad$ Symptoms $+2.517 *$ Limitation $+12.401 *$ Function-37.535

High quality of life $=2,717 *$ Symptoms $+3,396 *$ Limitation $+15,317 *$ Function-69,244

Table 3 Relevance of the classification variables. (Standardized coefficients of canonical discriminant functions)

\begin{tabular}{ll}
\hline Variables & Function I \\
\hline Symptom & 0.433 \\
Limitation & 0.181 \\
Emotional Function & 0.619 \\
\hline
\end{tabular}

The usefulness of these classification functions is to determine or predict the group to which a new individual will belong for which the values taken by the variables are known (Symptoms, Limitation and Emotional Function). When the data of these three variables are available for a new patient, it can be predicted to which group it belongs, evaluating the three functions and assigning it to the group for which a higher score has been obtained. The discriminant power of the variables Symptoms, Limitation and Emotional Function is very high, since $91.8 \%$ of the cases analyzed were classified correctly. Table 4 shows the successes and errors obtained in the classification made with the discriminant functions, specifically the group of predicted membership.

Table 4 Coefficients of the classification function

\begin{tabular}{llll}
\hline Variables & Quality life & Quality life & Quality life \\
& low & medium & high \\
\hline Symptom & 0.188 & 0.489 & 2.717 \\
Limitation & 2.324 & 2.517 & 3.396 \\
Emotional Function & 5.912 & 12.401 & 15.317 \\
(Constant) & -11.993 & -37.535 & -69.244 \\
\hline
\end{tabular}




\section{Discussion}

The results of our work indicate that the variable emotional function is the most relevant one on the quality of life of patients, an aspect coinciding with other studies that show that the symptoms that most affect HRQoL in children and adolescents is the pain and fatigue, interfering in the physical, emotional, social, academic aspects and in the general perception of well-being..$^{23,24}$ Other authors agree with the results of our analysis in the sense that asthma and allergic rhinitis not only produce a deterioration in the productivity and physical function of children and adolescents but also in the emotional aspects, affecting their closest environment, ${ }^{25,26}$ not being the determining factor in this regard the type of chronic disease suffered when its effects on the perception of HRQoL are compared..$^{27}$ A limitation of our study is that the number of students with asthma is undervalued, due to the methodology used to identify students with asthma through an open question and not having followed a questionnaire such as the "International Study of Asthma and Allergies in Childhood "(ISAAC) for the study in the school, a questionnaire used in other similar studies carried out in patients of these ages, although it should be noted that the symptoms and conclusions obtained in our study coincide with those indicated in the works previously reviewed and developed through the ISAAC questionnaire (Table 5). ${ }^{28,29}$

Table 5 Results of the classification

\begin{tabular}{lllll}
\hline & $\begin{array}{l}\text { Low quality } \\
\text { of life }\end{array}$ & $\begin{array}{l}\text { Average } \\
\text { life quality }\end{array}$ & $\begin{array}{l}\text { High } \\
\text { quality of } \\
\text { life }\end{array}$ & Total \\
\hline $\begin{array}{l}\text { Low quality } \\
\text { of life }\end{array}$ & $100 \%$ & $0 \%$ & $0 \%$ & $100 \%$ \\
$\begin{array}{l}\text { Average life } \\
\text { quality }\end{array}$ & $12.10 \%$ & $86.40 \%$ & $1.50 \%$ & $100 \%$ \\
$\begin{array}{l}\text { High quality } \\
\text { of life }\end{array}$ & $0 \%$ & $6.80 \%$ & $93.20 \%$ & $100 \%$ \\
\hline
\end{tabular}

\section{Conclusion}

We observe in students and teachers a low level of knowledge of asthma and attitudes that improve QL. Our study shows that there are no differences in the perception between boys and girls in the perception of QL. The perception of QL is not related to the attitudes towards asthma of the different groups analyzed. We think that the development of educational activities and educational measures in schools would be a basic factor to improve the quality of life and its perception by asthmatic children.

\section{References}

1. Working group of the Spanish Guide for the Management of Asthma Spanish Guide for the Management of Asthma (GEMA 4.0)2015.

2. Maldonado-Guzmán G. Quality of life in pediatric population aged 8 to 12 years with asthma. NURE Inv. 2007

3. Juniper E, Guyatt G, Feeny D, et al. Minimum skills required by children to complete health-related quality of life instruments for asthma: comparison of measurement properties. Eur Respir J. 1997;10(10):2285-2294.

4. Erhart M, Wetzel RM, Krügel A, et al. Effects of phone versus mail survey methods on the measurement of health-related quality of life and emotiona behavioral problems in adolescents. BMC Public Health. 2009;9:491-502.

5. Rabe KF, Adachi M, Lai CK, et al. Worldwide severity and control of asthma in children and adults: the global asthma insights and reality surveys. J Allergy ClinImmunol 2004;114:40-47.

6. Bateman ED, Boushey HA, Bousquet J, et al. Can guideline-defined asthma control be achieved? The Gaining Optimal Asthma ControL study. Am J Respir Crit Care Med. 2004;170(8):836-844.

7. Neffen H, Fritscher C, Schacht FC, et al. Asthma control in Latin America: the Asthma Insights and Reality in Latin America (AIRLA) survey. Rev Panam Salud Publica 2005;17(3):191-197.

8. Juniper EF, Guyatt GH, Feeny DH, et al. Measuring quality of life in children with asthma. Qual Life Res. 1996;5(1):35-46.

9. Juniper EF, Wisniewski ME, Cox FM, et al. Relationship between quality of life and clinical status in asthma: a factor analysis. Eur Respir J. 2004;23(2):287-291.

10. Bullinger M. Quality of life: Definition, conceptualization and implications: a methodologists view. Theor Surg. 19991;6:143-148.

11. Patrick D, Erickson P. Health Status and Health Policy: Quality of Life in Health Care Evaluation and Resource Allocation. New York: Oxford University Press. 1993. 478p.

12. Starfield B. Measurement of outcome: a proposed scheme. Milbank Mem Fund Q Health Soc. 1974;52(1):39-50.

13. Badia Llach X, Benavides Ruiz A, Rajmil Rajmil L. Instruments for measuring health-related quality of life in children and adolescents with asthma. An Esp Pediatr. 2001;54(3):213-221.

14. Juniper EF, Guyatt GH, Feeny DH, et al. Minimum skills required by children to complete health-related quality of life instruments for asthma: comparison of measurement properties. Eur Respir J. 1997;10(10):22852294.

15. Fitzclarence CA, Henry RL. Validation of an asthma knowledge questionnaire. J Paediatr Child Health. 1990;26(4):200-204.

16. Praena Crespo M, Lora Espinosa A, Aquino Llinares N, et al. A The Spanish version of the Newcastle Asthma Knowledge Questionnaire for parents of children with asthma (NAKQ). Transcultural adaptation and reliability analysis. An Pediatr (Barc). 2009;70(3): 209-217.

17. Chiou S, Jang C, Liao Y, et al. Health-related quality of life and cognitive out-comes among child and adolescent survivors of leukemia. Support Care Cancer. 2010;18(12):1581-1587.

18. Flokstra-de Blok B, van der Velde J, Vlieg-Boerstra B, et al. Health-related quality of life of food allergic patients measured with generic and diseasespecific questionnaires. Allergy. 2010;65(8):1031-1038.

19. Berkes A, Varni J, Pataki I, et al. Measuring health-related quality of life in Hungarian children attending a cardiology clinic with the Pediatric Quality of Life Inventory. Eur J Pediatr. 2010;169(3):333-347.

20. Chromá J, Slaný J. Quality of life of children with bronchial asthma disease. Cas Lek Cesk. 2011;150(12):660-664.

21. Badia X, García-Hernández G, Cobos N, et al. Validity of the Spanish version of the Pediatric Quality of Life Questionnaire for evaluating quality of life in asthmatic children. Med Clin (Barc). 2001;116(15):565-572.

22. Stahl E, Postmad S, Junipere F, et al. Health-related quality of life in asthma studies. Can we combine data from different countries? Pulm Pharmacol Ther. 2003;16(1):53-59.

23. Gold J, Mahrer N, Yee J, et al. Pain, fatigue, and health-related quality of life in children and adolescents with chronic pain. Clin J Pain. 2009;25(5):407-412. 
24. Petersen S, Hägglöf BL, Bergström EI. Impaired health-related quality of life in children with recurrent pain. Pediatrics. 2009;124(4):759-767.

25. Luna Mde F, Almeida PC, Silva MG. Prevalence and association of adolescent asthma and rhinitis of 13 and 14 years of Fortaleza, Ceará, Brazil. Cad.Saúde Pública. 2011;27(1):103-112.

26. Da Silva C, Da Silva T, Morales N, et al. Quality of life in children and adolescents with allergic rhinitis. Brazilian Journal Otorhinolaryngology. 2009;75:642-649.
27. Amaral CS, March Mde F, Sant'Anna CC. Quality of life in children and teenagers with atopic dermatitis. An Bras Dermatol. 2012;87(5): 717-723.

28. Lima W, Lima E, Ramos M, et al. Asthma and associated factors in adolescents aged 13 and 14 years old in São Luís, Maranhão, Brazil. Cad. Public Health 2012;28(6):1046-1056.

29. Sileyde C, Takano O, Moraes L, et al. Prevalence and risk factors for asthma in adolescents from 13 to 14 years of age in the city of Cuiabá, Mato Grosso, Brazil. Cad Public Health. 2012;28(4):689-697. 